\title{
Phase transitions in transportation networks with nonlinearities
}

\author{
C. H. Yeung and K. Y. Michael Wong \\ Department of Physics, The Hong Kong University of Science and Technology, Hong Kong, China \\ (Received 4 January 2009; revised manuscript received 21 May 2009; published 4 August 2009)
}

\begin{abstract}
We investigate a model of transportation networks with nonlinear elements which may represent local shortage of resources. Frustrations arise from competition for resources. When the initial resources are uniform, different regimes with discrete fractions of satisfied nodes are observed, resembling the Devil's staircase. We demonstrate how functional recursions are converted to simple recursions of probabilities. Behaviors similar to those in the vertex cover or close packing problems are found. When the initial resources are bimodally distributed, increases in the fraction of rich nodes induce a glassy transition, entering an algorithmically hard regime.
\end{abstract}

DOI: 10.1103/PhysRevE.80.021102

PACS number(s): 64.60.aq, 05.20.-y, 64.70.P-, 89.20.-a

The study of currents and flows in networks is one of the most important problems in physics and many other areas of application [1]. Besides electric circuits transporting electric currents, there are transportation networks, communications networks, hydraulic networks, mammalian circulatory systems and vascular systems in plants [2,3]. A unified approach to these problems is facilitated by the minimization of cost functions. For example, they may represent the dissipation energy (via Thomson's principle for electric currents) [1] or time delays in communications networks. There is a close relation between the flow patterns and the cost functions. For example, it was found that the flow pattern is treelike when the cost function is convex, but loopy otherwise [3]. These cost functions are continuous and often describe resistive flows. On the other hand, networks may contain nonlinear elements such as steplike penalties that may affect the flow distribution.

The inclusion of nonlinear elements can drastically modify the flow patterns in the network. Nonlinearities are often represented by cost functions that are discontinuous in the currents. This creates numerous choices in deciding the current-carrying links and the idle ones. As shown in this paper, the flow patterns can demarcate clusters of nodes receiving currents. Clusters formed by similar energetic considerations have been found to play an important role in disordered systems such as the random field Ising model (RFIM) [4], giving rise to the so-called Griffiths singularities and cascades of phase transitions [5]. As we shall see, similar behavior can also be found in nonlinear networks.

The origin of these interesting phenomena can be traced the presence of frustrations, which refers to the conflict between competing interaction energies in the system [6]. This connects such transportation networks with a broad class of network systems in which frustration is inherent, including the $K$ satisfiability [7], the graph coloring problem [8], and error-correcting codes [9]. Spin glasses, the prototype of frustrated systems, provide the statistical mechanics to study these systems [10].

Transportation networks consist of nodes with either surplus or shortage of resources, and an important problem is to distribute them to achieve a networkwide satisfaction with a minimum transportation cost [11]. This problem is important in load balancing in computer networks and network flow of commodities [12]. The focus of this paper is on the case where optimization of resources can proceed by penalizing nodes with shortages. In applications such as communications networks, such shortages can be detrimental to the performance. Hence it is interesting to consider steplike shortage costs, which give rise to unique behavior and physical picture absent in the previous models [11]. Frustrations arise from competition for resources among connected nodes. Numerous metastable states emerge, leading to typical glassy behavior.

Many NP-complete problems in computational complexity theory [13] exhibit glassy behavior. As we shall see, the main results in this paper are that changes in the shortage per node induce phase transitions to glassy behavior. A new feature in our model is a cascade of phase transitions resembling the Devil's staircase in the glassy phase, and the first cascade is similar to the vertex cover problem [14]. As a crucial difference from other NP-complete problems, the present problem involves continuous variables which complicate the analyses. We demonstrate how recursions of functions with continuous variables can be converted to simple recursions of probabilities.

Specifically, we consider a dilute network of $N$ nodes, labeled $i=1 \ldots N$. Each node is connected randomly to $c$ neighbors with the symmetric connectivity matrix $\mathcal{A}_{i j}=1,0$ for connected and unconnected node pairs respectively. Each node $i$ has initial resource or capacity $\Lambda_{i}$, randomly drawn from a distribution of $\rho\left(\Lambda_{i}\right)$. Positive and negative values of $\Lambda_{i}$ correspond to surplus and shortage of resources respectively. These resources can be transported through connected links. We denote by $y_{i j} \equiv-y_{j i}$ the current of resources from node $j$ to node $i$. The system minimizes the cost function

$$
E=\frac{u^{2}}{2} \sum_{i} \Theta\left(-\xi_{i}\right)+\sum_{(i j)} \frac{y_{i j}^{2}}{2} .
$$

$\xi_{i} \equiv \Lambda_{i}+\Sigma_{j} \mathcal{A}_{i j} y_{i j}$ is the final resources of node $i$, and $\Theta(x)$ $=1$ when $x>0$, and 0 otherwise. In the first term, each unsatisfied node yields a shortage cost of $u^{2} / 2$. The second term is the transportation cost of resources.

We first look for phase transitions in the model by numerical simulations. To formulate an algorithm, we introduce a variable $s_{i}= \pm 1$ for each node $i$ and restrict its resources by 


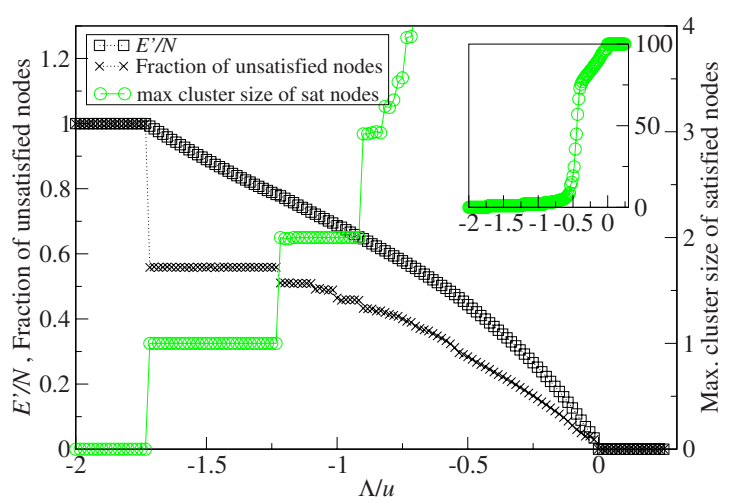

FIG. 1. (Color online) Simulation results of average energy per node, the fraction of unsatisfied nodes and the maximum cluster size of satisfied nodes after optimization, as a function of $\Lambda / u$. $E^{\prime}$ $=2 E / u^{2}$. Parameters: $c=3, N=100,100$ samples and 1000 flips. Inset: the maximum cluster size of satisfied nodes shown with a larger vertical scale.

$s_{i} \xi_{i} \geq 0$. Introducing Lagrange multipliers $\mu_{i}$ for the resource constraint, we minimize the Lagrangian

$$
L=\frac{u^{2}}{2} \sum_{i} \frac{1-s_{i}}{2}+\sum_{(i j)} \frac{y_{i j}^{2}}{2}+\sum_{i} \mu_{i} s_{i} \xi_{i}
$$

with the Kühn-Tucker conditions $\mu_{i} s_{i} \xi_{i}=0$ and $\mu_{i} \leq 0$. Optimizing $L$ with respect to $y_{i j}$, one obtains $y_{i j}=\mu_{j} s_{j}-\mu_{i} s_{i}$ and $\mu_{i}=\min \left[0,\left(\Lambda_{i}+\Sigma_{j} \mathcal{A}_{i j} \mu_{j} s_{j}\right) / s_{i} c\right]$. Given a particular set of $\left\{s_{i}\right\}$, we iterate these equations to find the corresponding set of $\left\{\mu_{i}\right\}$. The set of optimal $\left\{s_{i}\right\}$ is found by an approach similar to the GSAT algorithm [15], by comparing the Lagrangian in Eq. (2) for each choice of $\left\{s_{i}\right\}$.

We first consider networks with uniform capacity $\left(\Lambda_{i}=\Lambda\right.$ for all $i$ ). As shown in Fig. 1 for $c=3$, three phases can be identified: (1) unsatisfied phase for $\Lambda / u \leq-\sqrt{3}$, in which all the nodes are unsatisfied and $E / N=u^{2} / 2$; (2) partially satisfied phase for $-\sqrt{3}<\Lambda / u<0$, in which only some nodes are satisfied, and $0<E / N<u^{2} / 2$; (3) satisfied phase for $\Lambda / u$ $\geq 0$, in which all nodes are satisfied and $E / N=0$.

Unlike the relatively smooth decreasing trend of energy, the fraction of unsatisfied nodes is a discontinuous function of $\Lambda / u$, showing abrupt jumps at threshold values of $\Lambda / u$. The step size of the curve decreases as $\Lambda / u$ increases, and gradually becomes unresolvable by the numerical experiments. This resembles the Devil's staircase observed in the circle map and other dynamical systems [16]. These threshold values of $\Lambda / u$ mark the position at which certain configurations of the satisfied nodes become energetically stable.

To further confirm this picture, we measure the average maximum cluster size of satisfied nodes in the samples. Abrupt jumps of the cluster size are observed at the same threshold values as shown in Fig. 1. This indicates that new types of clusters of satisfied nodes are formed at each jump, as shown in Fig. 2 for $c=3$. The situation is similar to the cascades of phase transitions in RFIM due to the onsets of different clusters [5]. As shown in the inset of Fig. 1, the maximum cluster size increases drastically to $O(N)$ around

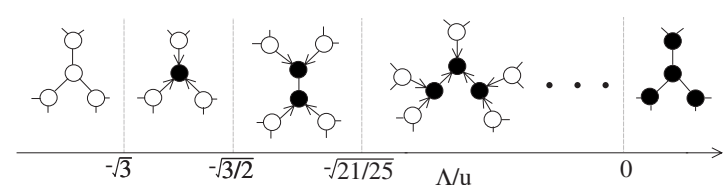

FIG. 2. The onset of different types of clusters of satisfied nodes for $c=3$, with filled and unfilled circles representing satisfied and unsatisfied nodes respectively.

$\Lambda / u \approx-0.5$. This corresponds to a percolation-like transition at which isolated clusters of satisfied nodes become connected.

We carry out the analysis using the Bethe approximation since the networks have a treelike structure locally. Messages are passed from the vertices of the subtrees to the next layer, resulting in a recursion relation of the messages. In the cavity approach, these messages are the cavity energy functions, denoted by $E_{j}\left(y_{i j}\right)$ for the energy of the tree terminated at a link from vertex $j$ to its ancestor $i$, when a current $y_{i j}$ is drawn from $j$ to $i$ [11]. They can be expressed in terms of the energies of its descendents $k=1, \ldots, c-1$,

$$
E_{j}\left(y_{i j}\right)=\min _{\left\{y_{j k}\right\}}\left\{\sum_{k=1}^{c-1} E_{k}\left(y_{j k}\right)+\frac{u^{2}}{2} \Theta\left[-\xi_{j}\left(y_{i j}\right)\right]+\frac{y_{i j}^{2}}{2}\right\},
$$

where $\xi_{j}\left(y_{i j}\right)=\Lambda_{j}+\sum_{k} y_{j k}-y_{i j}$.

In general, the iteration of Eq. (3) results in a distribution of the cavity energy functions, as shown in Fig. 3(a). However, in the regime $-\sqrt{c} \leq \Lambda / u \leq-\sqrt{c(c-1) /(c+1)}$, it can be shown analytically that there are $c$ functional forms of $E_{k}(y)$ forming a closed set of solutions to the recursion relation in Eq. (3). The three functions for $c=3$, referred to as the $u, s$, and $b$ states, are shown in Figs. 3(b)-3(d). Respectively, they correspond to states favoring unsatisfaction, satisfaction, and bistability, and have absolute minima at $y=0, y=\Lambda / c$, and both $y=0$ and $\Lambda / c$. Furthermore, numerical iterations of Eq. (3) starting with random $E_{k}(y)$ show that this set of solutions is stable. For $c>3$, the closed set consists of more states having absolute minima at $y=0$, and local minima at different energies at $y=\Lambda / c$, similar to the $u$ state in Fig. 3. As we shall see, the closure property of the $c$ states greatly simplifies Eq. (3). With the $u$ and $b$ states denoted as the $U$ state, and the $s$ state by the $S$ state, their recursion relations are given by an "SU recursion:" assign a vertex to an $S$ state if all its $c-1$ descendents are in the $U$ state, and to a $U$ state otherwise.
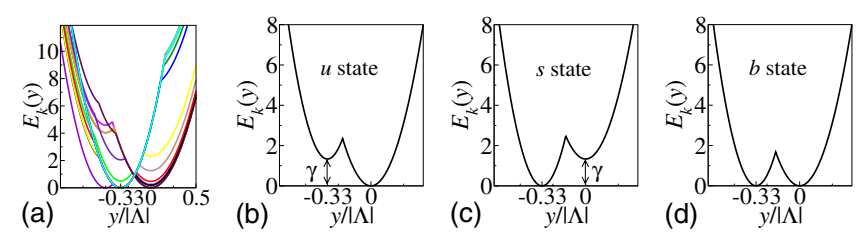

FIG. 3. (Color online) (a) A typical set of cavity energy functions $E_{k}(y)$ at $c=3, \Lambda / u=-1.22>-\sqrt{3 / 2}$. (b) - (d) The closed set of $E_{k}(y)$ at $c=3, \Lambda / u=-5 / 3$ with $\gamma=u^{2} / 2-\Lambda / 2 c$, corresponding to (b) the $u$ state, (c) the $s$ state and (d) the $b$ state. 

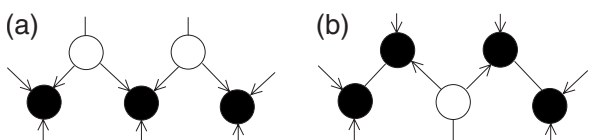

FIG. 4. (a) A five-node configuration composing of 3 isolated satisfied nodes (filled circles). It is stable when $\Lambda / u<-1$. (b) A five-node configuration composing of 2 two-node clusters. It replaces the configuration in (a) when $\Lambda / u>-1$.

The recursion rules imply that optimization is achieved by the close packing of satisfied nodes in the network, with the constraint that they do not form clusters. Hence we call the regime $-\sqrt{c}<\Lambda / u<-\sqrt{c(c-1) /(c+1)}$ the single-sat regime. This reduces the problem to the vertex cover problem [14]. Since there is at most one satisfied node at the end of each link, the maximization of the number of satisfied nodes is equivalent to the minimization of the covered set size in the vertex cover problem. Alternatively, the model can be considered as the close packing limit of a lattice glass model [17]. Both models exhibit glassy behavior, and phases with multiple metastable states are found therein, corresponding to the computationally hard phases.

The description of the glassy behavior can be approached by the replica analysis [10]. In the so-called replica symmetric (RS) ansatz, one assumes that the network behavior is dominated by a single ground state. However, we find that this ansatz is not stable in the entire single-sat phase. Instead, the network behavior is described by the so-called full replica symmetry-breaking (FRSB) ansatz, which assumes an infinite hierarchy of metastable states. Indeed, we have computed the optimized energy in the one-step RSB (1RSB) approximation, assuming that the optimized states with energy $E$ are distributed exponentially according to $\exp [N \Sigma(E)]$, where $\Sigma(E)$ is the so-called configurational entropy [18]. Futhermore, the 1RSB ansatz predicts that $\Sigma(E)$ exists up to an energy $E_{d}$ above the ground-state energy $E_{s}$, and the numerous metastable states prevent practical algorithms from converging to the true ground state, resulting in a dynamical transition. Specifically, we find that in the single-sat regime, $E / N=\Lambda^{2} / 6+f_{u}\left(u^{2} / 2-\Lambda^{2} / 6\right)$, where $f_{u}$ is the fraction of unsatisfied nodes. In the RS approximation, $f_{u}=0.545$, whereas in the 1RSB approximation, $f_{u}=0.549$ and 0.550 at its $E_{s}$ and $E_{d}$, respectively. Note that $f_{u}$ at the dynamical transition is close to the simulation result of $f_{u}=0.551$.

When $\Lambda$ increases above the single-sat regime, clusters of two satisfied nodes appear. This double-sat regime exists in the range $-\sqrt{3 / 2}<\Lambda / u<-\sqrt{21 / 25}$ for $c=3$. This is similar to the close packing limit of the Bethe glass model, in which each occupied site can have at most one occupied neighbor. However, the present model is richer in behavior, as indicated by the jumps in the fraction of unsatisfied nodes in the double-sat regime of Fig. 1. They mark the positions at which configurations increasingly dominated by two-node clusters become energetically stable, when $\Lambda$ increases. An example of such an energy switch is shown in Fig. 4. Indeed, such threshold values are expected at $\Lambda / u$ $=-\sqrt{(3 n-3) /(2 n-1)}$, where $n=2,3, \ldots$. Only two of those thresholds are visible in Fig. 1, probably due to the absence of configurations for larger $n$ in networks with $N=100$, and
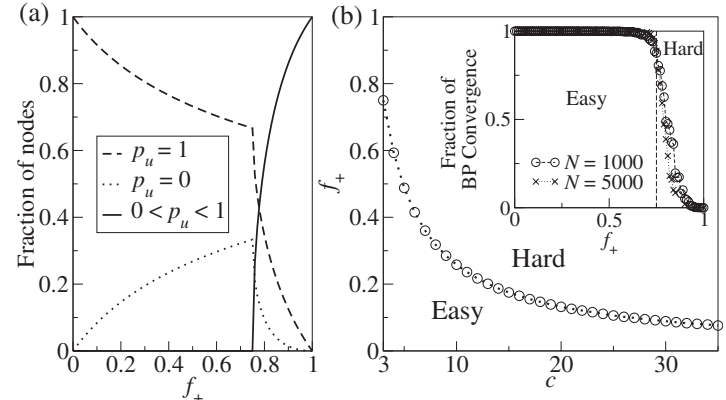

FIG. 5. (a) The fraction of nodes with $p_{u, k}=0,1$, and $0<p_{u, k}$ $<1$ for $c=3$. (b) The phase diagram. Inset: Simulation results of the fraction of messages from rich nodes with BP convergence on dilute networks with $c=3$.

the limitations of the search algorithm. Note that the doublesat regime may exhibit behaviors described by different RSB ansatz as $\Lambda / u$ varies. It is also interesting to study the possible change of RSB behavior as $\Lambda / u$ further increases up the Devil's staircase.

Next, we consider a simple case with quenched disorder of the node capacities. In this case, $\Lambda_{i}$ is drawn from a bimodal distribution, namely, $\Lambda_{i}=\Lambda_{ \pm}$with probabilities $f_{ \pm}$, where $\Lambda_{-} / u<-\sqrt{3}<\Lambda_{+} / u<-\sqrt{3 / 2}$ for $c=3$, and $f_{+}+f_{-}=1$. $\Lambda_{ \pm}$are referred to as the rich and poor nodes, respectively. The recursion relations for the rich nodes follow the $S U$ recursion, whereas the poor nodes are always in the $U$ state. Note that the end points of this range are $f_{+}=0$ and 1 , corresponding to the unsatisfied and partially satisfied phases, respectively. Since these two phases are in the RS and RSB regimes, respectively, we expect that there is a phase transition when $f_{+}$increases from 0 to 1 .

Applying the RS ansatz to the region of low $f_{+}$, we let $p_{u, k}$ be the probability that node $k$ is in the $U$ state. Its recursion relation can be written as

$$
p_{u, j}=\delta_{\Lambda_{j}, \Lambda_{-}}+\delta_{\Lambda_{j}, \Lambda_{+}}\left(1-\prod_{k=1}^{c-1} p_{u, k}\right),
$$

which corresponds to an intense simplification of Eq. (3). In the easy regime at low $f_{+}$, a stable fixed-point solution with all $p_{u, k}=0$ or 1 exists. The site average $\left\langle p_{u}\right\rangle$ can thus be obtained from the equation $\left\langle p_{u}\right\rangle=f_{-}+f_{+}\left(1-\left\langle p_{u}\right\rangle^{c-1}\right)$. In terms of algorithms, optimal network states in this regime can be obtained by the so-called belief propagation (BP) algorithm, initializing the messages to 0 or 1 .

The stability of the RS solution can be studied by considering the propagation of fluctuations $\left\langle\left(\delta p_{u, k}\right)^{2}\right\rangle$ under the recursion relation Eq. (4) [19]. This leads to the AlmeidaThouless (AT) stability condition, which reads

$$
(c-1) f_{+}\left\langle p_{u}^{2}\right\rangle^{c-2} \leq 1 .
$$

In the RS regime, $\left\langle p_{u}^{2}\right\rangle=\left\langle p_{u}\right\rangle$ since $p_{u, k}=0$ or 1 . This implies an AT transition at $f_{+}^{A T}=c^{c-2} /(c-1)^{c-1}$.

As shown in Fig. 5(a), free nodes with $0<p_{u, k}<1$ start to exist when $f_{+}>f_{+}^{\mathrm{AT}}$, analogous to the vertex cover [14] and graph coloring problems [8]. This characterizes the hard region with multiple states. 
As shown in the $f_{+}-c$ space in Fig. 5(b), the easy and hard phases exist below and above the AT line, respectively. In the large $c$ limit, $f_{+}^{\mathrm{AT}}$ approaches $e / c$. This result has an interesting connection with the vertex cover problem. Considering the cover set as the set of unsatisfied nodes, all links involving poor nodes are covered. The remaining links are those among the rich nodes, and the problem of minimizing the cover set size reduces to one that minimizes the subset size of covered nodes in the subnetwork of rich nodes. In the large $c$ limit, this subnetwork has a Poissonian connectivity distribution with an average $c f_{+}$. The result $c f_{+}^{\mathrm{AT}}=e$ agrees with the point of RS instability derived in [14].

The AT transition has implications on the algorithms. We consider the BP algorithm initialized with messages 0 and 1 . As shown in Fig. 5(b) inset, effectively all messages from rich nodes converge in the easy regime. However, a significant fraction of messages fluctuates between 0 and 1 above $f_{+}^{\mathrm{AT}}$, indicating the breakdown of the RS ansatz. Algorithmically, decimation procedures, such as those used in the sur- vey propagation (SP) algorithm [7], are required.

In summary, we have studied how nonlinearities affect the flow patterns in transportation networks. In the case of uniform capacity, phase transitions resembling the Devil's staircase reveal the cascades of clustered flow patterns. In the single-sat regime with a closed set of only a few cavity energy functions, the flow pattern has a correspondence with the vertex cover problem. Glassiness arises from the frustration in competitions for resources. In the case with quenched disorder of the capacities, an increase in the fraction of rich nodes induces a phase transition from an easy phase to a hard one, in which message-passing algorithms experience convergence problems. These features are relevant to general network optimization problems with nonlinear elements.

We thank David Saad and Jack Raymond for meaningful discussions and Patrick Lee for encouragement. This work is supported by the Research Grants Council of Hong Kong (Grants No. HKUST 603606, No. 603607, and No. 604008).
[1] P. G. Doyle and J. L. Snell, Random Walks and Electric Networks (Mathematical Association of America, Washington, DC, 1984).

[2] R. T. Rockafellar, Network Flows and Monotropic Optimization (Wiley, New York, 1984).

[3] J. R. Banavar, F. Colaiori, A. Flammini, A. Maritan, and A. Rinaldo, Phys. Rev. Lett. 84, 4745 (2000); M. Durand, ibid. 98, 088701 (2007); S. Bohn and M. O. Magnasco, ibid. 98, 088702 (2007); Z. Shao and H. Zhou, Phys. Rev. E 75, 066112 (2007).

[4] Y. Imry and S. K. Ma, Phys. Rev. Lett. 35, 1399 (1975).

[5] R. Bruinsma and G. Aeppli, Phys. Rev. Lett. 50, 1494 (1983); R. Bruinsma, Phys. Rev. B 30, 289 (1984).

[6] G. Toulouse, Commun. Phys. (London) 2, 115 (1977).

[7] M. Mézard and R. Zecchina, Phys. Rev. E 66, 056126 (2002).

[8] R. Mulet, A. Pagnani, M. Weigt, and R. Zecchina, Phys. Rev. Lett. 89, 268701 (2002); A. Braunstein, R. Mulet, A. Pagnani, M. Weigt, and R. Zecchina, Phys. Rev. E 68, 036702 (2003).

[9] H. Nishimori, Statistical Physics of Spin Glasses and Information Processing (Oxford University Press, Oxford, UK, 2001).

[10] M. Mézard, G. Parisi, and M. A. Virasoro, Spin Glass Theory and Beyond (World Scientific, Singapore, 1987).
[11] K. Y. Michael Wong and D. Saad, Phys. Rev. E 74, 010104(R) (2006); K. Y. Michael Wong and D. Saad, ibid. 76, 011115 (2007).

[12] S. Shenker, D. Clark, D. Estrin, and S. Herzog, Comput. Commun. Rev. 26, 19 (1996); R. L. Rardin, Optimization in Operations Research (Prentice Hall, Englewood Cliffs, NJ, 1998).

[13] M. R. Garey and D. S. Johnson, Computers and Intractability: A Guide to the Theory of NP-Completeness (Freeman, New York, 1979).

[14] M. Weigt and A. K. Hartmann, Phys. Rev. Lett. 84, 6118 (2000); Phys. Rev. E 63, 056127 (2001).

[15] B. Selman, H. Kautz, and B. Cohen, DIMACS Series in Discrete Mathematics and Theoretical Computer Science, 1996, Vol. 26, p. 521.

[16] R. L. Devaney, An Introduction to Chaotic Dynamical Systems (Addison-Wesley, Redwood City, CA, 1989).

[17] G. Biroli and M. Mézard, Phys. Rev. Lett. 88, 025501 (2001); O. Rivoire, G. Biroli, O. C. Martin, and M. Mézard, Eur. Phys. J. B 37, 55 (2004).

[18] M. Mézard and G. Parisi, Eur. Phys. J. B 20, 217 (2001).

[19] D. J. Thouless, Phys. Rev. Lett. 56, 1082 (1986). 